\title{
Carbon Ion Radiotherapy in the Management of Unusual Liposarcomas: A Case Report
}

\author{
VIVIANA VITOLO ${ }^{1}$, AMELIA BARCELLINI ${ }^{1}$, PIERO FOSSATI ${ }^{1}$, MARIA ROSARIA FIORE ${ }^{1}$, \\ BARBARA VISCHIONI ${ }^{1}$, ALBERTO IANNALFI ${ }^{1}$, ANGELICA FACOETTI ${ }^{1}$, MARIA BONORA ${ }^{1}$, \\ SARA RONCHI ${ }^{1}$, EMMA D'IPPOLITO ${ }^{1}$, RACHELE PETRUCCI ${ }^{1}$, GISELA VISELNER ${ }^{1}$, \\ LORENZO PREDA $^{1,2}$, MARIO CIOCCA $^{1}$, FRANCESCA VALVO $^{1}$ and ROBERTO ORECCHIA ${ }^{1,3}$ \\ ${ }^{1}$ National Center of Oncological Hadrontherapy (Fondazione CNAO), Pavia, Italy; \\ ${ }^{2}$ Department of Clinical-Surgical, Diagnostic and Pediatric Sciences, University of Pavia, Pavia, Italy; \\ ${ }^{3}$ European Institute of Oncology, Milan, Italy
}

\begin{abstract}
Background: Liposarcomas are the most common soft-tissue sarcomas in adulthood. Orbital and spermatic cord liposarcomas are uncommon and there is no consensus on their management. The treatment of choice is wide excision, which may be destructive and lead to unacceptable morbidity. When surgery is declined by patients and in recurrent disease, management can be challenging. We report two cases of liposarcoma treated with carbon ion radiotherapy at the National Center for Oncological Hadrontherapy (Fondazione CNAO) in Italy. Case Report: A woman with orbital liposarcoma and a man with spermatic cord liposarcoma were referred to our Center and accepted for carbon ion radiotherapy. The treatment was well tolerated and late toxicities were mild. Good local control was achieved in patients. Conclusion: In our experience, carbon ion radiotherapy is an effective and safe option, especially in cases of tumor at high risk for local relapse, in patients with multiple local recurrences, and in patients who refuse destructive surgery.
\end{abstract}

Liposarcomas (LPS) are the most common soft-tissue sarcomas in adulthood. The anatomic distribution of LPS varies widely, and they are commonly located in the extremities $(41 \%)$, retroperitoneum (19\%) and inguinal region (12\%) (1). Sarcomas of the genitourinary tract and of the head and neck are uncommon. Spermatic cord sarcomas

This article is freely accessible online.

Correspondence to: Angelica Facoetti, Fondazione CNAO, Strada Campeggi 53, Pavia, Italy. Tel: +39 0382078455, Fax: +39 0382078903,e-mail: angelica.facoetti@cnao.it

Key Words: Liposarcoma, carbon ion radiotherapy, spermatic cord, orbital. and orbital sarcomas are even more rare and their misdiagnosis frequently leads to incorrect management. Surgery remains the mainstay of treatment while chemotherapy and radiotherapy is proposed in selected cases. However, surgery with wide margins leads to unacceptable morbidity and for this reason, patients often refuse this approach. When surgery is declined by patients and in those with recurrent disease, the management can be challenging, as illustrated by the cases described herein.

Patients provided their consent to the publication regarding case details and associated images.

\section{Case Report}

Case 1: Dedifferentiated orbital LPS. A 41-year-old Caucasian woman complained of pain in her left eye. She had 10/10 visual acuity bilaterally with no limitation in motility. Brain magnetic resonance imaging (MRI) revealed an oval-shaped mass (maximum diameter: $1.3 \mathrm{~cm}$ ) arising anteriorly between the medial rectus muscle and the globe of eye, shaping and displacing the optic nerve laterally. T2-Weighted MRI showed an inhomogeneous high signal and was diagnostic for a cavernous hemangioma. The mass was removed and, surprisingly, pathology revealed a dedifferentiated LPS, grade 2 according to the French Federation of Comprehensive Cancer Centers grading system (2). Histologically, the tumor was composed of adipocytes including areas of dedifferentiation. Cells were loosely arranged, of varying sizes and the differentiated components were composed of spindle-shape cells that were immunohistochemically positive for murine double minute 2 ; acidic protein, $100 \%$ soluble in ammonium sulfate at neutral $\mathrm{pH}$ (S100); and cluster of differentiation 34 (CD34), but negative for desmin, SRY-related HMG-box 10 protein, epithelial membrane antigen, erythroblast transformation-specific-related-gene, and mucin 4. The proliferative index of the neoplastic cells was $15 \%$. 
After resection, the patient's visual acuity was preserved. The orbit MRI showed an inhomogeneous signal in the retrobulbar fat and a thickening of the medial rectus muscle (Figure 1A). Fluorodeoxyglucose $\left({ }^{18} \mathrm{~F}-\mathrm{FDG}\right)$ positronemission tomography (PET) was negative and ${ }^{11} \mathrm{C}$ methionine PET showed moderate uptake located on the medial side of the orbit. Imaging studies and physical examinations ruled out the possibility of a metastatic lesion of the orbit.

The patient refused the possibility of orbital exenteration followed by adjuvant radiotherapy. She was referred to our Center and accepted for carbon ion radiotherapy (CIRT). In preparation for the treatment, the patient was immobilized with a thermoplastic device in a supine position. A set of 2-mm-thick computed tomographic (CT) and contrastenhanced MR images was acquired for treatment planning, using the same immobilization device and rigidly registered each other in order to better outline target volumes and organs at risk.

The patient was treated with synchrotron-based scanning carbon ion beams (pencil beam scanning and spill-by-spill active energy variation). The dose prescribed was $73.6 \mathrm{~Gy}$ (relative biological effectiveness, RBE) in 16 fractions, 4.6 Gy (RBE) per fraction (4 fractions per week).

Syngo RT Planning treatment planning system (Siemens AG Healthcare, Erlangen, Germany), version C13, was used for plan optimization and calculation of RBE-weighted dose distributions according to the Local Effect Model version I, with the following parameters ( $\alpha \gamma: 0.1 \mathrm{~Gy}-1, \beta \gamma: 0.05 \mathrm{~Gy}-2$, Dt: 30 Gy, nuclear radius: $5 \mu \mathrm{m}$ ). Intensity-modulated particle therapy was employed for optimization. Patient set-up was verified at each treatment session by means of two independent custom verification systems, namely an infrared optical tracking system and a stereoscopic x-ray verification device (3-9). Six-degrees of freedom set-up correction vector was calculated and remotely applied to the treatment couch (Figure 1B).

Treatment was well tolerated and no interruption was needed. At the end of CIRT, the patient experienced grade 1 erythema, grade 1 conjunctivitis (according to Common Terminology Criteria for Adverse Events Version 4.0 (10) and a weak retro-bulbar pain that did not required medication. Follow-up with orbit MRI and ocular examination was carried out at our Center every 3 months and toxicities were recorded according CTCAE 4.0 grading score. She was followed-up for 28 months with no evidence of recurrence (Figure 1C). No high-grade late toxicities were reported except for a sporadic deficiency of the rectus muscles and grade 1 xerophthalmia. There was no evidence of radiation retinopathy or actinic optic neuropathy after CIRT and her visual acuity remained as it had been preoperatively.

Case 2: LPS of the spermatic cord. A 82-year-old man noticed a progressive painless swelling in the left inguinoscrotal region that was present for over a year. He noticed a more rapid growth in the weeks prior to consultation, with the swelling growing to $18 \mathrm{~cm}$ at presentation.

The patient was referred for a surgical option with a diagnosis of hydrocele. During surgery, the urologist found a mass arising not from testis but growing from the spermatic cord and descending into the scrotum around the testicles. A left orchidectomy was decided and performed. Pathology revealed a dedifferentiated LPS of the cord with rhabdomyosarcomatous differentiation and positive microscopical margins (R1). The patient underwent a second surgery to obtain clear surgical margins, confirmed by the subsequent histopathological examination which showed no residual neoplastic tissue. The patient was not given any adjuvant treatment and a close follow-up plan was recommended.

The patient remained asymptomatic throughout a followup period of 11.1 months, when MRI showed a scrotal tumor recurrence, sized $21 \times 15 \mathrm{~mm}$, confirmed by a subsequent biopsy. The surgical team decided to proceed with resection and intra-operative electron radiotherapy (total dose $10 \mathrm{~Gy}$ ) of the tumor bed to ensure a curative effect. Final pathology was consistent with a dedifferentiated LPS extending into the subcutaneous tissue with R1 margins.

At the uro-oncology tumor meeting, it was decided that adjuvant chemotherapy was not appropriate and thus patient was referred for inguinal radiotherapy. Two weeks later, the re-staging MRI revealed an early locoregional recurrence that was $32 \times 27 \mathrm{~mm}$ in size involving the omolateral corpus cavernosum (Figure 2A) and was confirmed by the subsequent FDG-PET.

The patient was then referred to our Center for particle therapy evaluation and he was accepted for CIRT. He was treated with synchrotron-based scanning carbon ion beams (pencil beam scanning and spill-by-spill active energy variation). For treatment planning, the patient was immobilized in the supine position with a custom thermoplastic mask and a set of CT images of $2 \mathrm{~mm}$ thickness and a contrast-enhanced MRI were performed and rigidly registered against each other in order to better outline target volumes and organs at risk.

A CE-marked Syngo RT Planning treatment planning system (Siemens AG Healthcare, Erlangen, Germany), version C13, was used for plan optimization and calculation of RBEweighted dose distributions according to the Local Effect Model version I $(3-9,11)$. The dose prescribed was $76.8 \mathrm{~Gy}$ (RBE) in 16 fractions, $4.8 \mathrm{~Gy}$ (RBE) per fraction (4 fractions per week) for an overall treatment time of 28 days (Figure 2B). An intensity-modulated particle therapy was employed as plan optimization strategy. Patient set-up was verified at each treatment session by means of two independent custom verification systems, namely an infrared optical tracking system and a stereoscopic x-ray verification device (11). Sixdegrees of freedom set-up correction vector was calculated and remotely applied to the treatment couch. 

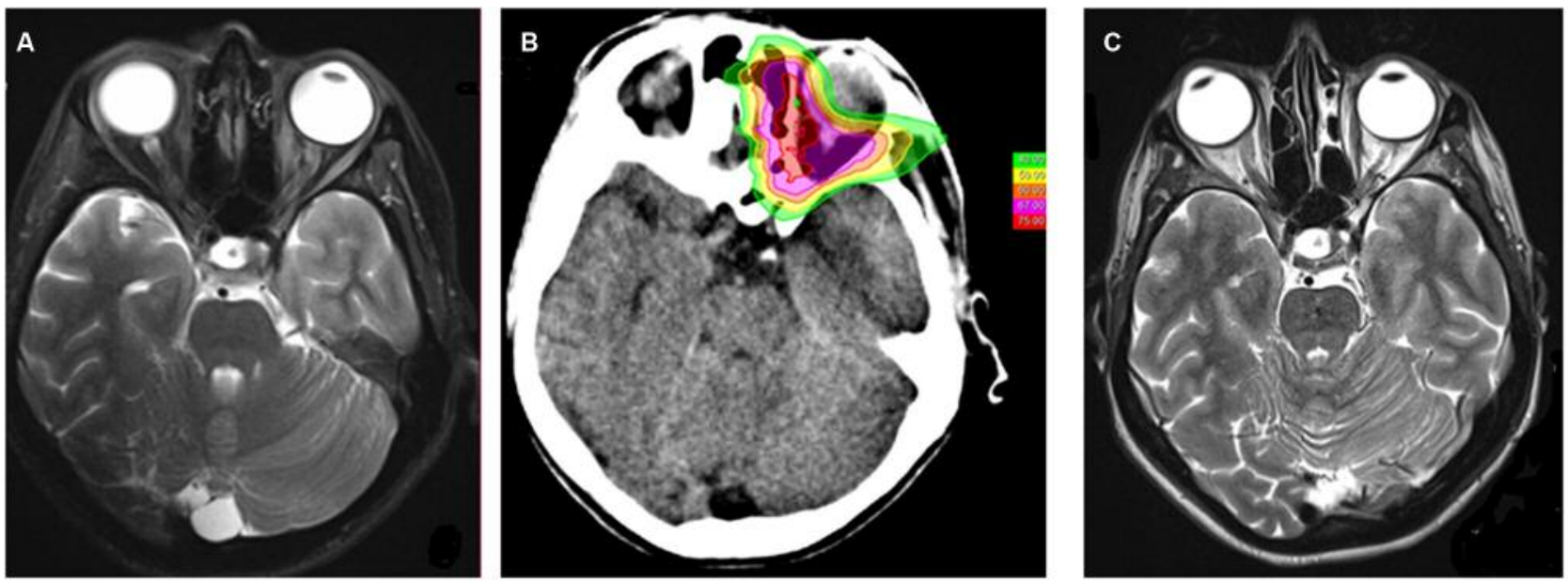

Figure 1. Orbital liposarcoma. A: Magnetic resonance imaging before carbon ion radiotherapy showed an inhomogeneous signal in the retrobulbar fat and a thickening of the medial rectus muscle. B: Plan treatment: the maximum dose constraint was maintained at $\leq 60 \mathrm{~Gy}$ (relative biological effectiveness, RBE) to the optic chiasm; the mean dose constraint was maintained at $<50 \mathrm{~Gy}(\mathrm{RBE})$ to the globe, at $<40 \mathrm{~Gy}(\mathrm{RBE})$ to the anterior left chamber. No constraints were applied to left optic nerve and to the left retina. C: Magnetic resonance imaging after carbon ion radiotherapy (28 months after the treatment): no evidence of recurrence.
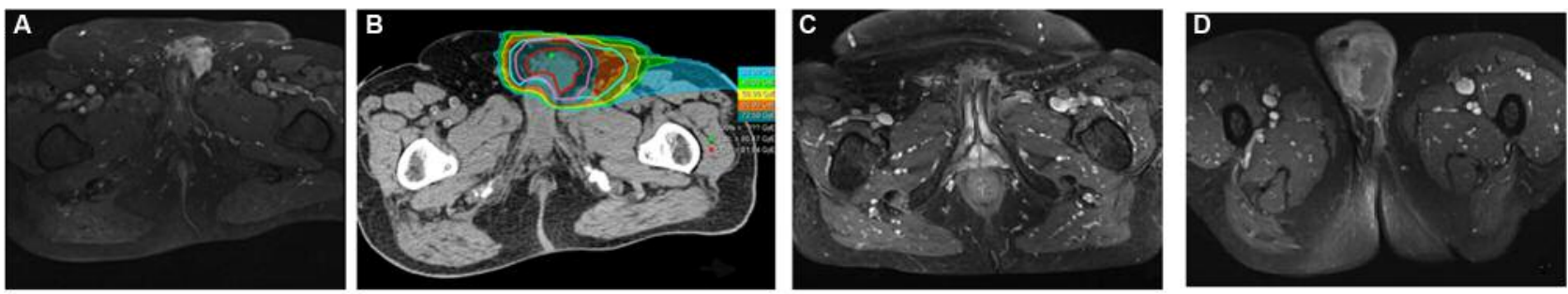

Figure 2. Spermatic cord liposarcoma. A: Magnetic resonance imaging at diagnosis. B. The dose distribution: the maximum dose constraint was maintained at $\leq 70$ Gy (relative biological effectiveness) to the urethra. C and D: Local response and scrotal relapse after a progression-free period of 28 months.

Treatment was well tolerated and no interruption was needed. At the end of CIRT, the patient had experienced only a grade 1 erythema according to CTCAE 4.0. No late toxicity was reported except for local grade 1 fibrosis (CTCAE 4.0). Unfortunately the patient experienced relapse in the scrotum after a progression-free period of 28 months (Figure 2C and D).

\section{Discussion}

To our knowledge, there are no reports concerning CIRT in the management of orbital and spermatic cord LPS. Compared to photons, CIRT has a superior dose conformity which is even better than modern radiotherapy techniques such as stereotactic body irradiation or intensity-modulated radiation therapy/volumetric-modulated arc radiotherapy. CIRT also has greater biological advantages, due to a higher linear energy transfer of heavy ions, resulting in better local tumor control and lesser adverse effects on normal tissues. Hence, CIRT may be an effective treatment for patients with LPS who refuse destructive surgery (Case 1) or in patients with recurrent disease (Case 2).

Despite a large amount of adipose tissue, the orbit is an extraordinarily rare location for lipogenic tumor, even more so for primary or secondary LPS. To the best of our knowledge, only 27 cases of primary orbital LPS have been reported and only a few cases of metastatic orbital LPS are documented in literature. Only six cases of dedifferentiated orbital LPS have been documented worldwide so far; including this case, the age of the patients ranged from 23 to 56 years, with a predilection for women (ratio $\mathrm{M}: \mathrm{F}=6: 1$ ) contrary to other LPS that are more frequent in men (13-21).

By having a mass effect, orbital LPS creates signs and symptoms such as proptosis, ptosis, diplopia, swelling, optic 
neuropathy, pain and loss of visual acuity. Clinical examination and MRI of the orbit are essential to study the mass, its local extension and its relationship with surrounding structures. However, no radiographic features are pathognomonic for its diagnosis, which remains histological. The diagnosis might also be very challenging, especially in small biopsy samples, however modern immunohistochemical techniques may increase diagnostic precision. LPS is known for its high tendency to spread locoregionally and to recur; even if treatment of orbital LPS is controversial, the cornerstone of treatment remains surgery. Destructive surgery is recommended for invasive, dedifferentiated and recurrent tumors and adjuvant radiation therapy can be used when surgery is not complete. Conservative surgery followed by radiotherapy is indicated for small, well-defined and easily accessible tumors. Due to its rarity, there is no consensus about the use of chemotherapy or immunotherapy (11-21).

Indeed, full excision seems to be the best approach to enable better local control and improved survival. Nevertheless orbital LPS cannot be completely excised without functional or aesthetic damage and for these reasons, patients often refuse surgery. CIRT for head and neck cancer has already been proven a promising treatment, particularly for patients with radioresistant or inoperable tumors (22). To the best of our knowledge this report is the first focusing on CIRT, to demonstrate good local control (disease-free survival of 28 months) and an excellent preservation rate of the ipsilateral eyeball.

The second case study presented in this report was LPS of the spermatic cord with high propensity for recurrence. Although unusual, the spermatic cord is the most commonly involved urological site of genitourinary sarcomas. Due to its rarity, there are no consensus treatment protocols but in order to obtain satisfying locoregional control, surgery performed through a wide en bloc excision of the mass with radical orchiectomy, excision of the ipsilateral scrotum and high spermatic cord ligation is recommended. Therefore, iliac and femoral vessels must be exposed, examined and, in selected cases, can be excised and reconstructed. Unless there is evidence of tumor, lymph node dissection or inguinal adjuvant radiotherapy are not indicated because of unclear benefits, the rarity of lymph node metastasis and postoperative/post-actinic complications $(23,24)$.

Negative microscopic margins are essential since local recurrence is more frequent when the resection is, as in our case, incomplete. Indeed, in patients who underwent surgery for LPS of the spermatic cord, the status of surgical margins proved to be significantly associated with disease-specific survival ( $p=0.043$, 5-year survival: $100 \%$ in patients with negative margins and 94\% with R1) (23).

However, resection margins are almost invariably close and either second resection or adjuvant radiotherapy are advised. Radiotherapy should also be provided in the case of presumed high risk of tumor relapse, such as in those with high-grade tumor and previous multiple local recurrences. As in LPS of the orbit, adjuvant chemotherapy with/without radiotherapy should be administered on a case-by-case basis because there are insufficient data to evaluate their effectiveness.

In 2014, Radaelli et al. published a series of 82 patients with spermatic cord sarcoma (33\% well-differentiated LPS, $29 \%$ dedifferentiated LPS, $21 \%$ leiomyosarcoma and $17 \%$ miscellanea of sarcomas) and reported a rate of 5-year-local recurrence of $26 \%$ (95\% confidence interval 14-42\%) for the whole series and a median time to recurrence for patients who experienced a local relapse of 21 months (range $=3-101$ months). Moreover, in that series, patients with a history of recurrent tumors experienced greater local relapse as compared to those with primary tumors (5-year local recurrence rate: $37 \%, 95 \%$ confidence interval $=18-65 \mathrm{vs}$. $22 \%$, 95\% confidence interval=10-43\%) even if the difference was not statistically significant $(p=0.129)(23)$.

In this subgroup of patients, to which our patient belongs, the management is challenging and CIRT might be an effective and safe option for evaluation due to its higher relative biological effectiveness associated with greater linear energy transfer that many give superior probability of achieving tumor control with a lower post-radiation morbidity.

\section{Conclusion}

Primary dedifferentiated LPS of the orbit and of spermatic cord are rare entities and their management remains controversial. In summary, in two patients with atypical LPS, CIRT was applied to the lesions while minimizing high doses to the organ at risk. In our experience, CIRT proved to be effective, feasible and safe, with a negligible toxicity. Thus we believe that CIRT must be considered as an effective and safe option in cases of tumor at high risk for local relapse, in patients with multiple local recurrences and in patients who refuse destructive surgery.

\section{References}

1 Wibmer C, Leithner A, Zielonke N, Sperl M and Windhager R: Increasing incidence rates of soft tissue sarcomas? A populationbased epidemiologic study and literature review. Ann Oncol 21: 1106-1111, 2010.

2 Fletcher CDM Rydholm A, Singer S, Sundaram M and Coindre JM: Soft tissue tumours: Epidemiology, clinical features, histopathological typing and grading. In: World Health Organization Classification of Tumours. Pathology and Genetics of Tumours of Soft Tissue and Bone. Fletcher CDM, Unni KK and Mertens F (eds.). Lyon, lARC Press, pp. 12-18, 2002.

3 Fossati P, Molinelli S, Matsufuji N, Ciocca M, Mirandola A, Mairani A, Mizoe J, Hasegawa A, Imai R, Kamada T, Orecchia $\mathrm{R}$ and Tsujii H: Dose prescription in carbon ion radiotherapy: a planning study to compare NIRS and LEM approaches with a clinically-oriented strategy. Phys Med Biol 57: 7543-7554, 2012. 
4 Molinelli S, Magro G, Mairani A, Matsufuji N, Kanematsu N Inaniwa T, Mirandola A, Russo S, Mastella E, Hasegawa A, Tsuji H, Yamada S, Vischioni B, Vitolo V, Ferrari A, Ciocca M, Kamada T, Tsujii H, Orecchia R and Fossati P: Dose prescription in carbon ion radiotherapy: How to compare two different RBEweighted dose calculation systems. Radiother Oncol 120: 307312,2016

5 Krämer M and Scholz M: Treatment planning for heavy-ion radiotherapy: calculation and optimization of biologically effective dose. Phys Med Biol 45: 3319-30, 2000

6 Lomax A: Intensity modulated methods for proton therapy. Phys Med Biol 44: 185-205, 1999.

7 Mirandola A, Molinelli S, Vilches Freixas G, Mairani A, Gallio E, Panizza D, Russo S, Ciocca M, Donetti M, Magro G Giordanengo S and Orecchia R: Dosimetric commissioning and quality assurance of scanned ion beams at the Italian National Center for Oncological Hadrontherapy. Med Phys 42(9): $5287-$ 5300, 2015

8 Molinelli S1, Mairani A, Mirandola A, Vilches Freixas G, Tessonnier T, Giordanengo S, Parodi K, Ciocca M and Orecchia R: Dosimetric accuracy assessment of a treatment plan verification system for scanned proton beam radiotherapy: Oneyear experimental results and Monte Carlo analysis of the involved uncertainties. Phys Med Biol 58: 3837-3847, 2013.

9 Pella A, Riboldi M, Tagaste B, Bianculli D, Desplanques M, Fontana G, Cerveri P, Seregni M, Fattori G, Orecchia R and Baroni G. Commissioning and quality assurance of an integrated system for patient positioning and setup verification in particle therapy. Technol Cancer Res Treat 13: 303-314, 2014.

10 National Cancer Institute: Common Terminology Criteria for Adverse Events (CTCAE), Version 4.0. Available at: http://evs.nci.nih.gov/ftp1/CTCAE/About.html. Accessed on 27 September 2018.

11 Rossi S: The National Centre for Oncological Hadrontherapy (CNAO): Status and perspectives. Phys Med 31: 333-351, 2015

12 Giordanengo S, Garella MA, Marchetto F, Bourhaleb F, Ciocca M, Mirandola A, Monaco V, Hosseini MA, Peroni C, Sacchi R, Cirio R and Donetti M. The CNAO dose delivery system for modulated scanning ion beam radiotherapy. Med Phys 42: 263$75,2015$.

13 Zhang JX, Ma JM and Wang NL: Dedifferentiated orbital liposarcoma: A case report. Int J Ophthalmol 4: 452-453, 2011.

14 Cai YC, McMenamin ME, Rose G, Sandy CJ, Cree IA and Fletcher CD: Primary liposarcoma of the orbit: A clinicopathologic study of seven cases. Ann Diagn Pathol 5: 255-266, 2001.
15 Saeed MU, Chang BY, Atherley C, Khandwala M, Merchant D and Liddington M. A rare diagnosis of dedifferentiated liposarcoma of the orbit. Orbit 26: 43-45, 2007.

16 Stiglmayer $\mathrm{N}$, Jandrokovicć $\mathrm{S}$, Miklicć $\mathrm{P}$ and Hutinec $\mathrm{Z}$ : Atypical lipoma: Well-differentiated liposarcoma of the orbit with dedifferentiated areas. Orbit 22: 311-316, 2003.

17 Torrado CS, Zaldibar NM, Velasco-Benito V and FernándezHermida R: Primary orbital liposarcoma. J Craniofac Surg 22(3): 1139-1141, 2011.

18 Wagle AM, Biswas J, Subramaniam N and Mahesh L: Primary liposarcoma of the orbit: A clinicopathological study. Orbit 18 : 33-336,1999.

19 Costas A, Castro P and Munuoz JM: Primary orbital liposarcoma: case report and review of the literature. Int J Oral Maxillofac Surg 30: 170-172, 2001.

20 Kraus DH, Dubner S, Harrison LB, Strong EW, Hajdu SI, Kher $\mathrm{U}$, Begg C and Brennan MF. Prognostic factors for recurrence and survival in head and neck soft-tissue sarcomas. Cancer 74 : 697-702, 1994

21 Al-Qahtani AA, Al-Hussain H, Chaudhry I, El-Khamary S and Alkatan HM: Primary liposarcoma of the orbit: a report of two cases. Can J Ophthalmol 42: 481-483, 2007.

22 Mizoe JE, Hasegawa A, Jingu K, Takagi R, Bessyo H, Morikawa T, Tonoki M, Tsuji H, Kamada T, Tsujii H, Okamoto Y; Organizing Committee for the Working Group for Head Neck Cancer. Results of carbon ion radiotherapy for head and neck cancer. Radiother Oncol 103: 32-37, 2012.

23 Radaelli S, Desai A, Hodson J, Colombo C, Roberts K, Gourevitch D and Gronchi A: Prognostic factors and outcome of spermatic cord sarcoma. Ann Surg Oncol 21: 3557-3563, 2014.

24 Chalouhy C, Ruck JM and Moukarzel M: Current management of the spermatic cord: A case report and review of the literature. Mol Clin Oncol 6: 438-440, 2017.
Received November 29, 2018

Revised December 7, 2018

Accepted December 11, 2018 